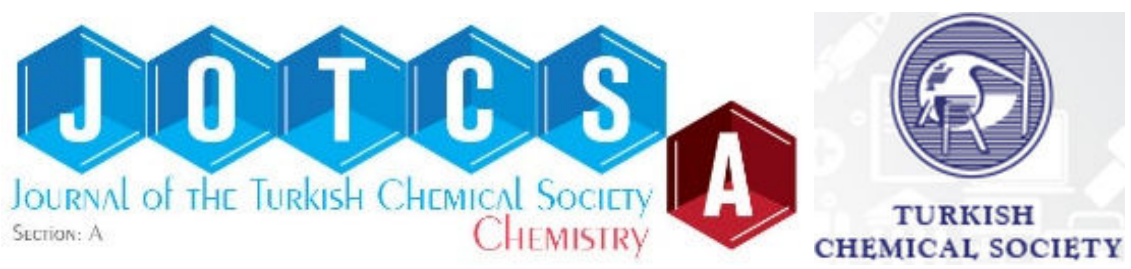

(This article was presented to the 28th National Chemistry Congress and submitted to JOTCSA as a full manuscript)

\title{
Misens Device as a New Automated Biosensing Platform Based on Real-Time Electrochemical Profiling (REP)
}

\author{
Yildiz Uludag* \\ Bioelectronics Devices and Systems Group - UEKAE - BILGEM - The Scientific and \\ Technological Research Council of Turkey (TUBITAK), 41470 Gebze/Kocaeli, Turkey.
}

\begin{abstract}
In various fields like health, environmental control, food security, and military defense, there is an increasing demand for on-site detection, fast identification, and urgent response which bring about the necessity to employ laboratory detection procedures on standalone automatic devices. In response to that, TUBITAK BILGEM's Bioelectronic Devices and Systems Group has been developing portable and fully automated biosensor devices using optical and electrochemical biosensor detection techniques. Here we describe a new integrated and fully automated lab-on-a-chip based biosensor device 'MiSens'. The key features of the MiSens include a new electrode array, an integrated microfluidic system, and real-time amperometric measurements during the flow of enzyme substrate. While simple protocols can be controlled from the LCD display on the device, other main device control procedures can be run wireless by a tablet/PC using the MiCont ${ }^{T M}$ software developed by the team. For the device, a new plug and play type sensor chip docking station has been designed that with one move it enables the formation of $a \sim 7-10 \mu \mathrm{L}$ capacity flow cell on the electrode array with the necessary microfluidic and electronic connections. The MiSens device has been developed by our multi-disciplinary team by integrating and automatising the earlier developed sensing platform REP ${ }^{\mathrm{TM}}$ (Real-time Electrochemical Profiling). The performance of the MiSens device has been tested using cyclic voltammetry and amperometry tests and the results were compared with an of the shelf potentiostat.
\end{abstract}

Keywords: Real-time electrochemical profiling; biosensor; amperometry.

Submitted: July 14, 2016. Revised: September 08, 2016. Accepted: September 30, 2016.

Cite this: Uludağ Y. Misens Device as a New Automated Biosensing Platform Based on Real-Time Electrochemical Profiling (REP). JOTCSA. 2016;3(3):403-16.

DOI: $10.18596 /$ jotcsa.65921.

*Corresponding author. E-mail: Yildiz.uludag@tubitak.gov.tr, Tel: 02626481910. 


\section{INTRODUCTION}

Biosensors are analytical devices that comprise a biological recognition element, a suitable transducer, and an appropriate data processing system (1-3). While one of the main application of the biosensors are in the research and development for biomolecule interaction analysis or drug discovery, it is also possible to design biosensors for end users in order to perform analytical measurements for environmental monitoring, quality assurance in agriculture/food and medical diagnostics (4). The number of biosensorrelated articles has exceeded 9000 / year; search on the term "biosensor*" for 2015 yields 9226 articles, using the Web of Knowledge, 8 times more than that of the year 2000. Compared with the numerous publications and patents available since the early 1990 's, the commercialisation of the miniaturised biosensor devices for end users has significantly lagged behind the research output. $(2,5)$. However, in recent years due to the advances in microelectronics, nanotechnology, bioengineering, and especially the microfluidic technologies, new on-site or point-of-care biosensor devices started to appear in the diagnostics market indicating a significant change (6). Currently, testing for in vitro diagnostics is typically performed in centralised laboratories using large automated analysers in hospitals $(7,8)$. Similarly, tests for environmental monitoring and quality control of food/agriculture products typically performed in centralised laboratories using expensive laboratory equipment and trained personnel (2, 9-12). To eliminate sample transportation, to reduce the time spent between the sample acquisition to the results and hence for a rapid response to an emergency, fully automated on-site testing devices are promising tools. Portable biosensor devices connected through the Internet of Things may change the way we perform the analytic measurements not only in healthcare but also for other application areas of the biosensors. Therefore, for on-site testing, a biosensing platform needs to be able to work automatically with minimum user interference. The tasks that need to be automated may include sample preparation, separation, detection, waste collection, and data analysis. While it is reasonably easy to manipulate fluids using the bench top biosensor devices using automated pipetting and robotic arms, for portable devices that deals with microlitres or smaller amounts of liquids it constitutes a challenge. Recent advances in microfluidics have enabled the miniaturisation of the devices and multiplex testing of a range of analytes (13-15). Advanced micro fabrication techniques have facilitated integration of sensing functionalities with microfluidics on the same biochip enabling automated systems (14). To address the need in automated and on-site testing of analytes, a new electrochemical biosensor device has been developed by TUBITAK 
BILGEM that relies of Real-time Electrochemical Profiling (REPTM) and novel electrode array based biochips.

\section{MATERIALS AND METHODS}

\section{Materials}

Phosphate buffered saline (PBS, $0.01 \mathrm{M}$ phosphate buffer, $0.0027 \mathrm{M}$ potassium chloride and $0.137 \mathrm{M}$ sodium chloride, $\mathrm{pH}$ 7.4) tablets, mercaptoundecanoic acid (MUDA), spectrophotometric grade ethanol, horseradish peroxidase (HRP), 3,3',5,5'tetramethylbenzidine (TMB) ready to use reagent (includes $\mathrm{H}_{2} \mathrm{O}_{2}$ ), potassium ferrocyanide $\left(\mathrm{K}_{4}\left[\mathrm{Fe}(\mathrm{CN})_{6}\right]\right)$ and $\mathrm{KCl}$ were purchased from Sigma-Aldrich (Poole, UK).

\section{Electrochemical Analysis}

An integrated and fully automated electrochemical biosensor has been designed and fabricated (MiSens ${ }^{\mathrm{TM}}$ V01; Figure 1-A) by BİLGEM-Bioelectronic Devices and System Development Group. MiSens device is controlled wireless using a tablet/PC with the developed MiCont ${ }^{T M}$ software (TUBİTAK-BİLGEM, Kocaeli, Turkey). Cyclic voltammetry $(\mathrm{CV})$ tests were performed using $1 \mathrm{mM}$ potassium ferrocyanide solution in $1 \mathrm{M} \mathrm{KCl}$. The MiSens biosensor chip includes an electrode array that is fabricated on a $10 \times 20 \mathrm{~mm}$ glass slide. Each array consist of 8 working electrodes ( $d=1 \mathrm{~mm}$ ) with shared $\mathrm{Au}$ counter and quasi-reference electrodes (Error! Reference source not found.-B) (16). The design of the electrodes was formed on the glass slide by means of a Fine Metal Mask made of a laser cut patterned stainless steel and Au metal was deposited on the wafer using an electron beam evaporator (Torr EB-4P, New Windsor, US). Before the application of $\mathrm{Au}(200 \mathrm{~nm})$, a $20 \mathrm{~nm}$ Ti layer is applied on to the wafer as an intermediary adhesive layer to increase the adhesion between the Au and the glass. For easy handling of the sensor chip, a holder has been designed and fabricated from poly(methyl methacrylate) (PMMA) (Figure 1-B). 


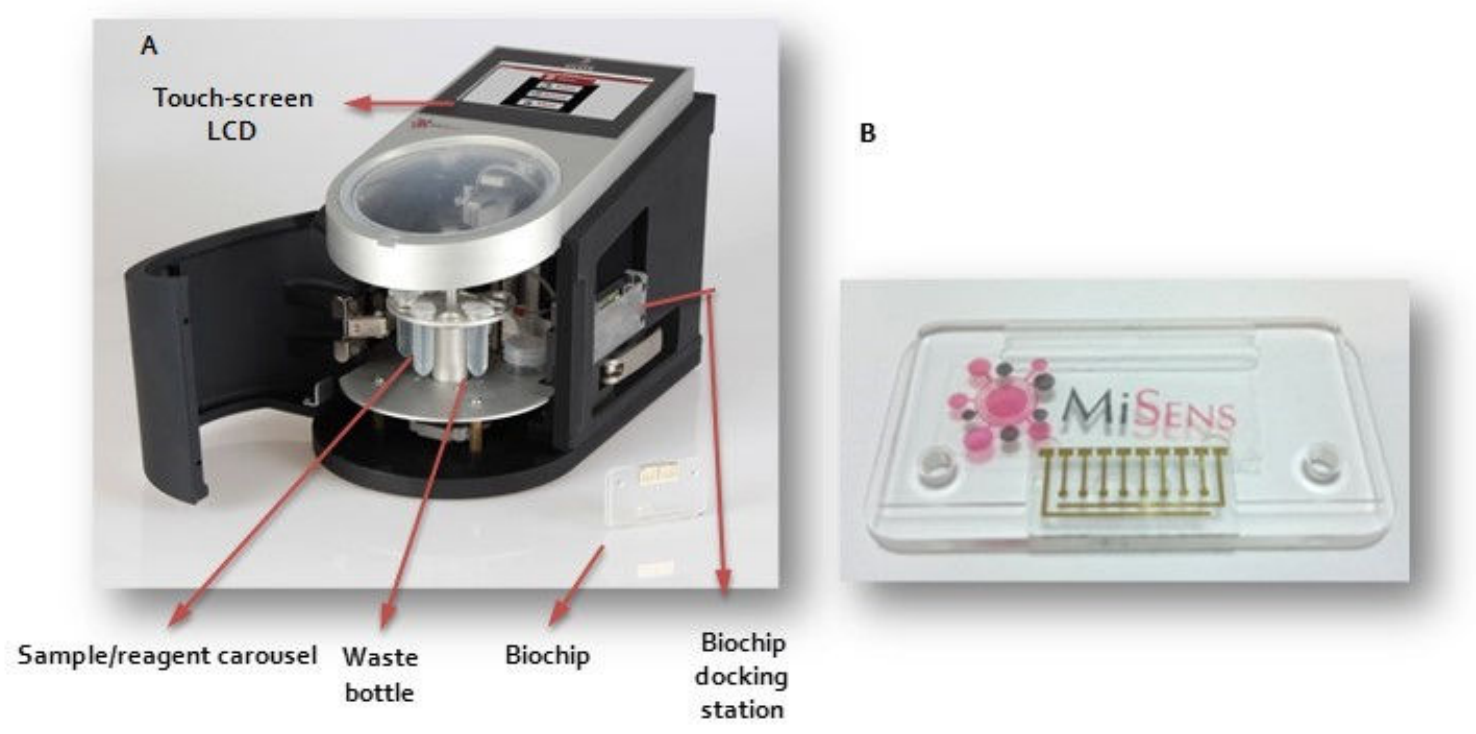

Figure 1. Fully integrated and automated MiSens biosensor device $(A)$ and its biochip (working electrode diameter $1 \mathrm{~mm}$ ) (B).

For comparison purposes, the tests has been replicated using an off-the-shelf screen printed electrodes (SPE) (Dropsens, Oviedo, Spain). Using the SPEs, cyclic voltammetry and amperometric measurements were performed with a MicroStat 8000 Electrochemical Analyser with the general purpose electrochemical software Dropview 1.4 (Dropsens, Oviedo, Spain). The electrochemical analyser and the purpose built shielded cables enable simultaneous electrochemical measurements of 8 electrodes. Cyclic voltammetry (CV) tests were performed using $1 \mathrm{mM}$ potassium ferrocyanide solution in $1 \mathrm{M} \mathrm{KCl}$.

\section{Biochip Surface Modification}

Surface modification of the SPE and MiSens biochips with a self-assembled monolayer (SAM) has been achieved by plasma cleaning of the arrays and later immersing in an ethanolic solution of 2 mM MUDA for overnight. Later, the electrode arrays were rinsed with ethanol and water. After drying with nitrogen stream, the arrays were vacuumpacked and stored at $+4^{\circ} \mathrm{C}$ till use.

\section{Enzymatic Assay}

For the MiSens assay, enzyme assays were performed by mixing HRP enzyme and TMB substrate, then injecting $150 \mu \mathrm{L}$ of this solution to the biochip at $55 \mu \mathrm{L} / \mathrm{min}$. The real-time 
amperometric measurements were recorded by the MiCont ${ }^{\mathrm{TM}}$ software (TUBITAK BILGEM) for analysis. The chronoamperometric responses obtained at $-0.1 \mathrm{~V}$ potential at the $150 \mathrm{~s}$ of the measurements were used as assay response. For the SPE assays, $50 \mu \mathrm{L}$ of the enzyme-substrate solution has been dropped on the inidividual electrodes and the amperometric signal has been recorded using the Dropview 1.4 software. The chronoamperometric responses obtained at $-0.1 \mathrm{~V}$ potential at the $90 \mathrm{~s}$ of the measurements were used as assay response. For the MiSens assays, 8 data points, for the SPE assays, 3 data points were used to obtain the mean and standard deviation of the results. The limit of detection (LOD) was calculated as the signal obtained from the assays that is equivalent to 3 times the standard deviation of the signals obtained from the blank standards.

\section{RESULTS AND DISCUSSION}

\section{Device Integration}

The MiSens device has been developed by TUBITAK BILGEM's multi-disciplinary team by integrating and automatising the earlier developed sensing platform REPTM (17). The key features of this platform include a new biochip (18), an integrated microfluidic system and real-time amperometric measurements during the flow of enzyme substrate. In earlier studies, an electrode array has been developed that utilises shared reference and counter electrodes to minimise the size of the sensor. Later, a new plug and play type sensor chip docking station has been designed. With one move, the chip docking station enables the formation of $a \sim 7 \mu \mathrm{L}$ capacity flow cell on the electrode array with the necessary microfluidic and electronic connections. The mechanical parts of the MiSens device include biochip docking station, a pump, microfluidic tubing connected sample pick up needle, sample/reagent carousel, and a waste bottle. The electronic parts of the device include an LCD display, multiplexed potentiostat, a digital control circuit and wired/wireless communication interface. MiCont ${ }^{\mathrm{TM}}$ software enables user to create a list of assay steps that forms the test protocol of the MiSens device (Figure 2). The user can save and re-use the protocol created when needed. During data acquisition, the MiCont software shows the electrochemical measurements real time. 


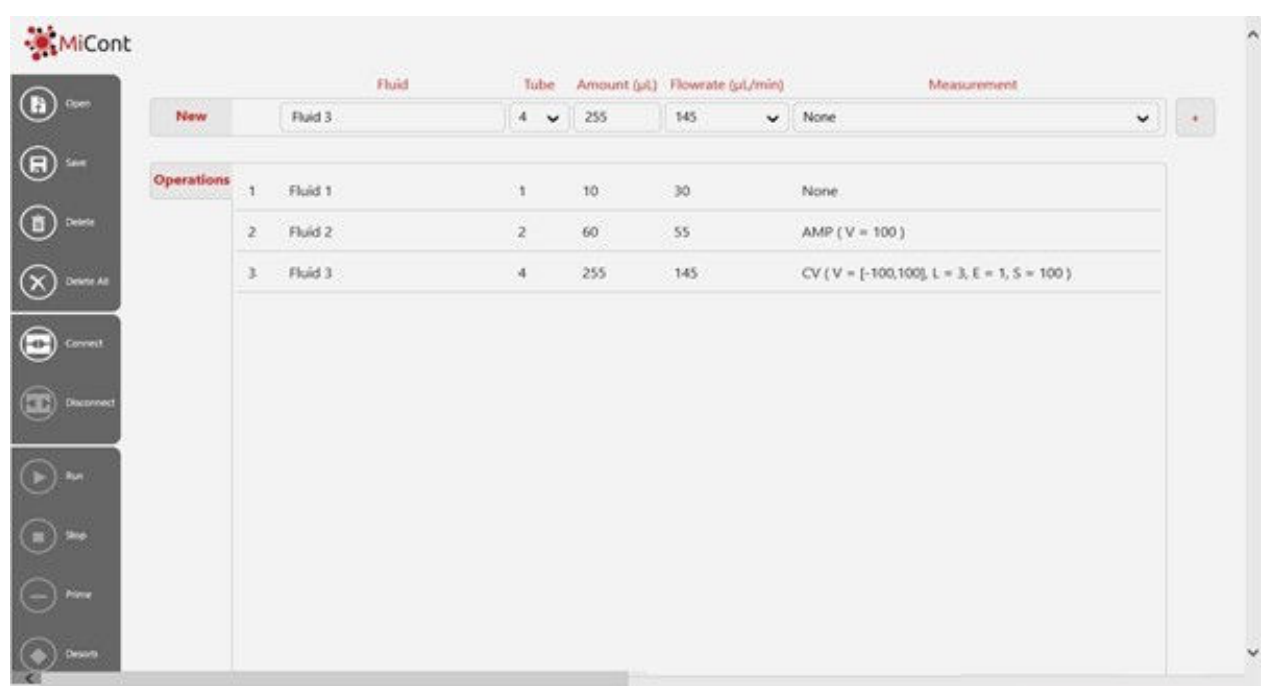

Figure 2. The MiCont ${ }^{\mathrm{TM}}$ software developed by the team that enables fast and easy assay protocol formation prior to automated assay run.

\section{Sensor Surface Characterization}

To investigate the electrochemical behaviour of the designed electrode arrays, cyclic voltammetry technique has been utilised and voltammograms were recorded for a model electroactive species, potassium ferricyanide $(19,20)$. Application of alkanethiols on a gold electrode surface forms a self-assembled monolayer and hence insulates the electrode surface $(21,22)$. By comparing the cyclic voltammograms of a bare gold electrode and SAM coated gold electrode, it is possible to investigate the coverage of the monolayer. Cyclic voltammograms of bare Au and SAM coated MiSens biochip and SPE were obtained using $\mathrm{K}_{4}\left[\mathrm{Fe}(\mathrm{CN})_{6}\right]$ as the electroactive marker. As it could be seen from the Figure $3 \mathrm{~A}-\mathrm{C}$, the oxidation and reduction peaks of $\mathrm{K}_{4}\left[\mathrm{Fe}(\mathrm{CN})_{6}\right]$ was visible and there was a larger redox area between positive and negative potential sweeps when cyclic voltammetry performed on bare gold surfaces. Whereas, the redox area between positive and negative potential sweeps of the SAM coated sensor chips were smaller in SPE, and non-existed in MiSens biochip. The SPE surface is formed using an ink containing Au and polymer, this surface shows a rough surface characteristic. While rough surface results in a larger electroactive area, the existence of polymer rather than pure Au results in lower coverage of SAM layer as seen from the cyclic voltammogram. However, MiSens biochips are made using pure $A u$ that is sputtered on a flat glass slide. This surface is not as rough as the SPE, however as the electrode is formed using pure Au, the SAM coverage of the surface is higher as seen from the cyclic voltammogram (Figure $3 B$ ). 
The surface coverage $(\theta)$ of the SAM can be calculated by comparing the charge (Q) during reduction/oxidation of cyclic voltammetry at a monolayer covered electrode with respect to a bare gold electrode (Equation 1$)(23,24)$.

$$
\theta=1-\left(Q_{S A M} / Q_{\text {bare gold }}\right)
$$

The surface coverage of the SAM-coated MiSens biochip and the screen printed electrode has been calculated using Equation 1 as $0.96 \pm 0.03$ and $0.54 \pm 0.06$, respectively.
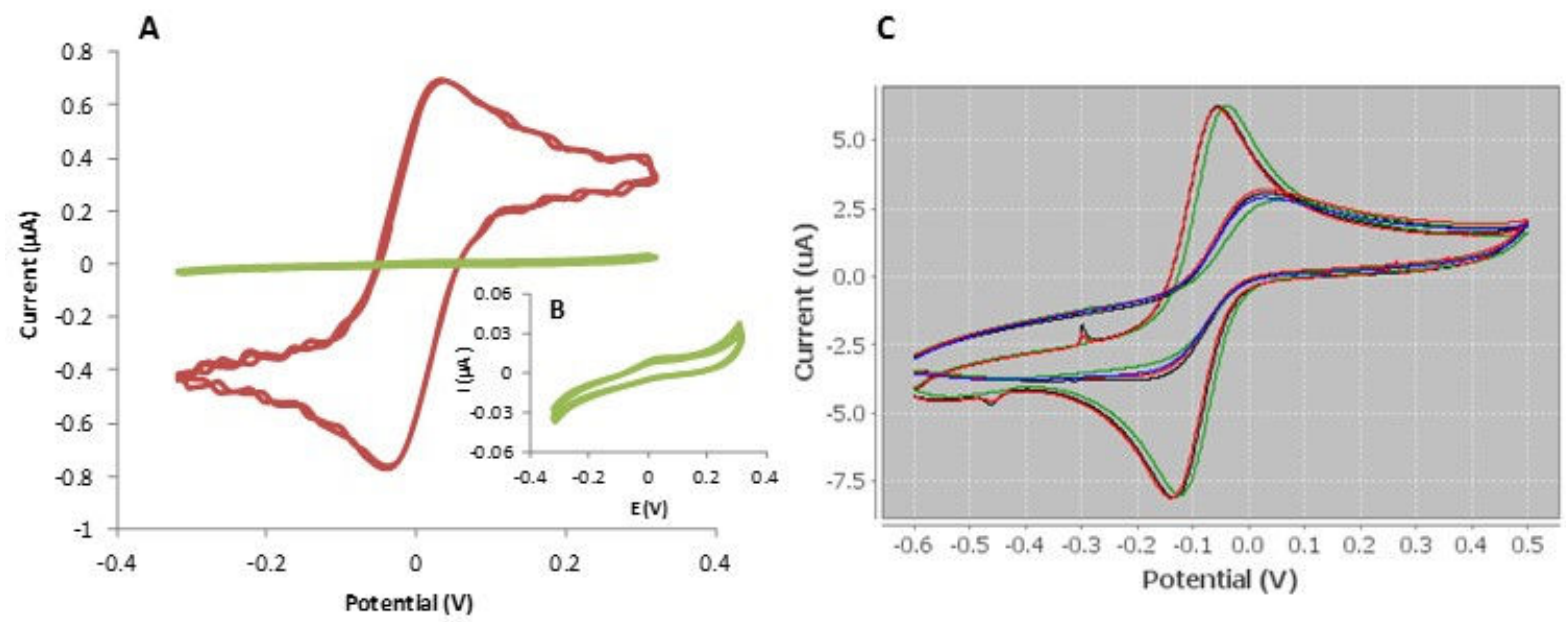

Figure 3. Cyclic voltammetry has been performed with $1 \mathrm{mM} \mathrm{K} 4\left[\mathrm{Fe}(\mathrm{CN})_{6}\right] / \mathrm{KCl}$ at 100 $\mathrm{mV} / \mathrm{s}$ can rate, using bare and MUDA coated MiSens biochips $(\mathrm{A}, \mathrm{B})$ and SPE (C).

\section{Enzymatic activity measurement of HRP}

Horseradish peroxidase (HRP) is an enzyme label widely used in immunoassays. The enzymatic activity of HRP and its substrate TMB (3,3',5,5'-tetramethylbenzidine) can be determined by measuring the absorbance in the visible region, by fluorescence or by electrochemistry (25). During the enzyme-linked immunosorbent assay (ELISA), after the addition of the TMB substrate on to the enzyme-bound microwell plate, the reaction is allowed to take place around 15 minutes before stopping the reaction and measuring the colour formation by a spectrophotometer. The aim of 15 minutes reaction is to increase the colour formation and hence obtain a reasonable optical detection signal. As the electrochemical measurements are more sensitive than the optical detection, 15 minutes reaction may not be needed for the measurement. During the Real-time Electrochemical Profiling (REP) assay, the HRP and TMB mixture is injected on to the electrode arrays of the MiSens biochip and a real-time amperometric signal is obtained at $-0.1 \mathrm{~V}$ potential (Figure 4 ). 

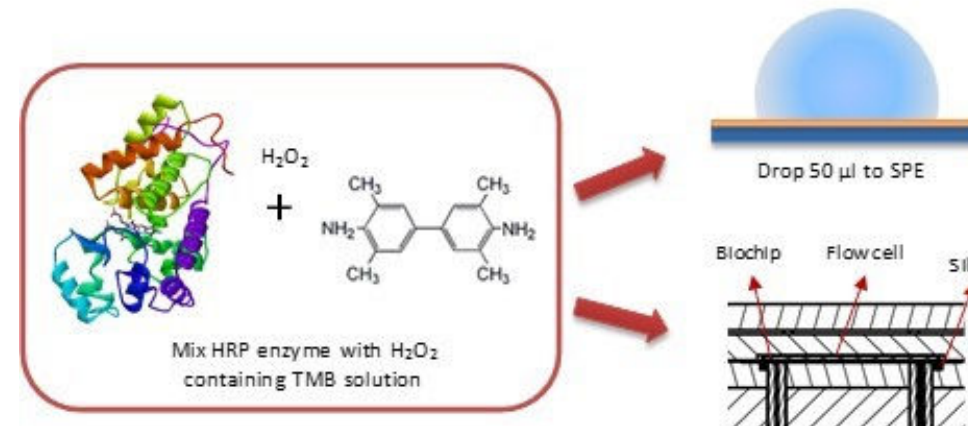

Drop $50 \mu$ ul to SPE

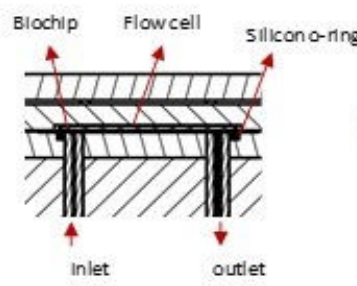

Inject $150 \mu$ l to MiSens biochip

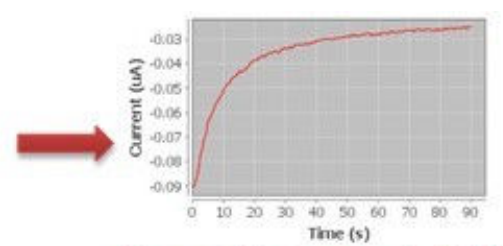

Amoerometric measurements at $-0.1 \mathrm{~V}$

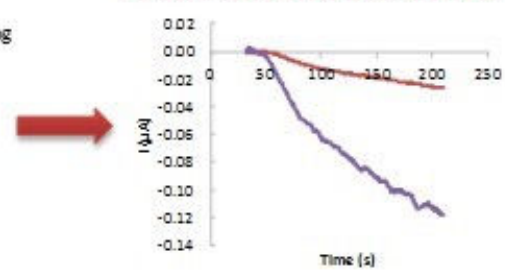

Time (s)

Figure 4. The chemical reaction of TMB in the presence of the HRP enzyme has been measured as an amperometeric signal using both SPE and MiSens biochip.

For the HRP enzymatic activity measurement assays, initially plasma cleaned bare Au SPE and MiSens biochips were coated with a SAM layer. The Au surface of the electrodes were coated with a self assembled monolayer (MUDA) to prevent the adsorbance of HRP on the electrode array surface and also to mimick a recognition element (antibody, DNA etc) immobilised electrode, in other words an electrode with a reduced electrochemically active area. Later, the enzyme assays were performed by mixing varying concentrations of HRP with a fixed concentration of TMB reagent, then injecting to the flow cell containing electrode arrays for MiSens, or dropping the solution on to the SPE surface. Later the chronoamperometric responses obtained at $-0.1 \mathrm{~V}$ potential has been analysed (See Figure 4). In Figure 5, the raw data obtained from the enzyme assay using Misens biochips and device is shown. In Figure 6-A, the amperometric signals obtained from both SPE and MiSens biochip are compared. As seen from this figure, the results obtained from both SPE and MiSens biochips were comparable to each other with coefficient of determination of 1.0 and 0.98 , respectively. Although similar amperometric results have been obtained from both sensor chips, as the electroactive area of both chips differ, a considerable difference was observed when current density is calculated. As MiSens biochips have $1 \mathrm{~mm}$ diameter with respect to $4 \mathrm{~mm}$ diameter of SPE chips, the current density of MiSens biochips are 14.4 times higher than the SPE (Figure 6-B, 
Table 1).

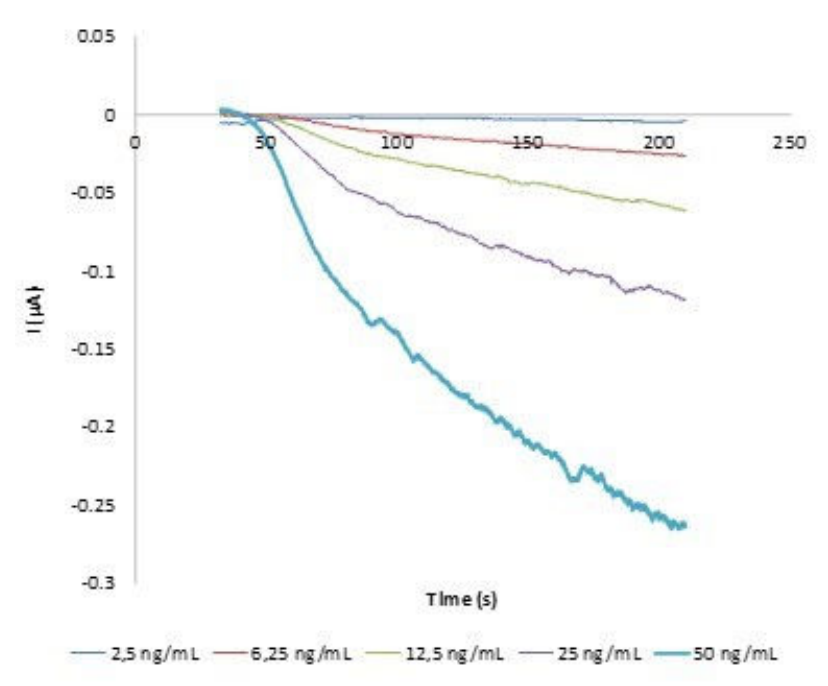

Figure 5. Enzymatic activity measurement assays were performed by mixing varying concentrations (2.5 - $50 \mathrm{ng} / \mathrm{mL}$ ) of horseradish peroxidase (HRP) and TMB reagent, then injecting to the SAM coated electrodes in a flow cell for chronoamperometric measurements. The current measurements were taken at $-0.1 \mathrm{~V}$ potential. The plot shows the raw data obtained from the MiSens device.

A

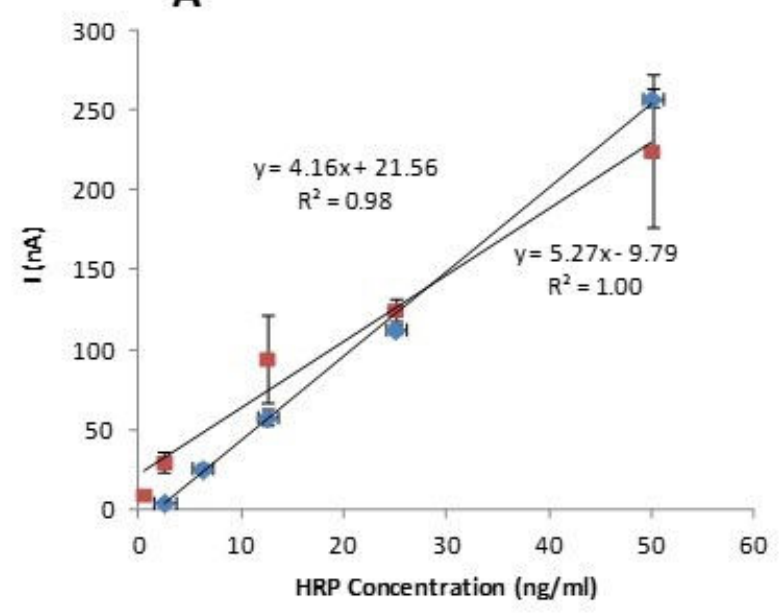

II Au-SPE(MUDA coated); Dropsens uStat

- MiSensbiochip (MUDA coated) and device
B

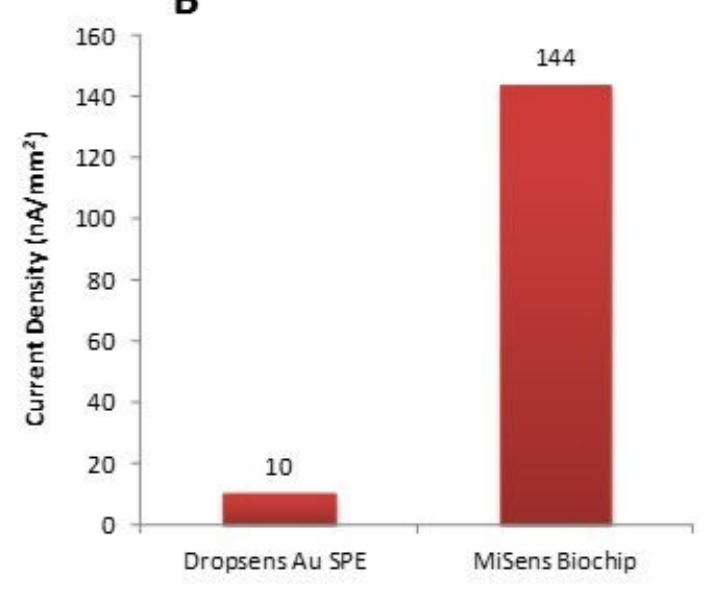

Figure 6. A) The amperometric response from the enzymatic reaction of HRP and TMB has been plotted with respect to HRP concentration for MiSens and SPE electrodes. B) The current response obtained from the chronoamperometry results after the addition of $25 \mathrm{ng} / \mathrm{mL}$ HRP and TMB reagent were used to assess the current density on MiSens biochip and Au SPE. 
Table 1. The curreny density results for SPE and MiSens biochip.

\begin{tabular}{cccc}
\hline MUDA-coated & $\begin{array}{c}\text { Response for } \mathbf{2 5} \mathbf{~ n g} / \mathbf{m L} \\
\text { HRP }(\mathbf{n A})\end{array}$ & Area $\left(\mathbf{m m}^{\mathbf{2}}\right) *$ & $\begin{array}{c}\text { Current density } \\
\left(\mathbf{n A} / \mathbf{m m}^{\mathbf{2}}\right)\end{array}$ \\
\hline $\begin{array}{c}\text { Dropsens Au SPE } \\
(\mathrm{d}=4 \mathrm{~mm})\end{array}$ & 125 & 12.56 & 9.98 \\
\hline $\begin{array}{c}\text { MiSens Biochip }(\mathrm{Au}) \\
(\mathrm{d}=1 \mathrm{~mm})\end{array}$ & 113 & 0.79 & 143.75 \\
\hline
\end{tabular}

*The area calculation does not take into account the roughness of the SPE chip. If that is also considered, the current density of the SPE chip will further decrease. The MiSens chip is produced by sputtering Au on a glass slide surface, hence its roughness is minimal with respect to the SPE chips.

The HRP and substrate reaction incubation time have direct effect to the detection limit of the assay and usually ranges from a few minutes to an hour. While in almost all studies minimum 15 minutes of HRP reaction time is allowed, in the current study the aim was to minimise the assay time since the biosensor device MiSens has been designed for on-site or point of care detection. As can be seen from Table 2, even without any incubation, a detection limit of $3.4 \times 10^{-11} \mathrm{M}$ has been achieved for the HRP enzyme activity using the MiSens biosensor device. Here, we have shown that MiSens device can be used as a flow injection analysis system for the detection of HRP enzymatic activity, hence an indication that it can be utilised as an electrochemical ELISA reader. In addition to that the easily programmable automated flow injection system of the device enables the injection of multiple analytes to the sensor surface in the required order hence immunoassays can be performed on the electrode surface directly with ease (26). 
Table 2. HRP enzyme activity measurements using different electrochemical setups.

\begin{tabular}{|c|c|c|c|c|c|c|}
\hline 1 System & 2 Electrode type & $\begin{array}{l}3 \text { Electrode } \\
\text { diameter }\end{array}$ & 4 Microfluidics & $\begin{array}{l}5 \text { Incubation before } \\
\text { measurement }\end{array}$ & $\begin{array}{ll}6 & \text { Detection } \\
& \text { Limit } \\
7 & \text { (HRP } \\
& \text { Concentratio } \\
& \text { n) }\end{array}$ & $\begin{array}{l}8 \text { Refere } \\
\text { nce }\end{array}$ \\
\hline $\begin{array}{l}\text { MiSens biochip \& } \\
\text { device }\end{array}$ & Au electrode & $1 \mathrm{~mm}$ & $\begin{array}{l}\text { Automated flow } \\
\text { injection analysis }\end{array}$ & None & $3.4 \times 10^{-11} \mathrm{M}$ & $\begin{array}{c}\text { Current } \\
\text { work }\end{array}$ \\
\hline SPE \& Potentiostat & Au SPE & $4 \mathrm{~mm}$ & $\mathrm{x}$ & None & $1.1 \times 10^{-11} \mathrm{M}$ & $\begin{array}{l}\text { Current } \\
\text { work }\end{array}$ \\
\hline $\begin{array}{l}\text { Disk electrode and } \\
\text { potentiostat }\end{array}$ & $\begin{array}{l}\text { Glassy carbon } \\
\text { electrode }\end{array}$ & $3 \mathrm{~mm}$ & Flow injection analysis & $30 \mathrm{~min}$ & $2.6 \times 10^{-12} \mathrm{M}$ & (27) \\
\hline $\begin{array}{l}\text { Disk electrode and } \\
\text { potentiostat }\end{array}$ & $\begin{array}{l}\text { Glassy carbon disk } \\
\text { working electrode }\end{array}$ & $3 \mathrm{~mm}$ & Flow injection analysis & $15 \mathrm{~min}$ & $8.5 \times 10^{-14} \mathrm{M}$ & $(25)$ \\
\hline SPE \& potentiostat & Carbon SPE & $4 \mathrm{~mm}$ & Flow injection analysis & $30 \mathrm{~min}$ & $2 \times 10^{-14} \mathrm{M}$ & $(28)$ \\
\hline
\end{tabular}




\section{CONCLUSIONS}

The recent advances in electronics, bioengineering and lab-on-a-chip technologies results in the development of new generation bioanalytics devices for out-of-the-laboratory and on-site testing. By combining the device development capabilities of BILGEM TUBITAK with bioengineering and nanotechnology, the BILGEM Bioelectronics group has developed a new fully automated electrochemical biosensor device, MiSens and biochips. The performance of this new electrochemical device and biochips have been compared to a commercial potentiostat with its SPE chips. The results show that the smaller footprint of the MiSens biochip ( $\mathrm{d}=1 \mathrm{~mm}$ ) and integrated microfluidics enables similar amperometric responses to the HRP-TMB enzymatic assay with respect to the SPE ( $d=4 \mathrm{~mm})$. However, when the current obtained with respect to the sensor surface area is compared, the current density of the MiSens chips has been found 14.4 times higher, showing the effectiveness of the biochip design and the microfluidic system. The fully automated format of the MiSens device and its microfluidic system enables electrochemical measurements to be performed with ease, hence provides a very effective tool for research and development of new biosensing assays. In the future, MiSens device has also a potential to be used for on-site testing of environmental / food toxins, and for biodefense.

\section{ACKNOWLEDGMENTS}

The project is supported by the BILGEM - TUBITAK (The Scientific and Technological Research Council of Turkey) (grant no: 100121). We gratefully acknowledge Bioelectronic Devices and System Group from BILGEM - TUBITAK for their contribution to the design and fabrication of the biosensor device (MiSens) and the biochips.

\section{REFERENCES}

1. Lowe C. An introduction to the concepts and technology of biosensors. Biosensors. 1985;1(1):316.

2. D'Orazio P. Biosensors in clinical chemistry-2011 update. Clinica Chimica Acta. 2011;412(1920):1749-61. DOI: $10.1016 /$ j.cca.2011.06.025.

3. Keusgen M. Biosensors: new approaches in drug discovery. Naturwissenschaften. 2002;89(10):433-44. DOI: 10.1007/s00114-002-0358-3. 
4. Turner APF. Biosensors: sense and sensibility. Chemical Society Reviews. 2013:3184-96. DOI: 10.1039/C3CS35528D.

5. Luong JHT, Male KB, Glennon JD. Biosensor technology: Technology push versus market pull. Biotechnology Advances. 2008;26(5):492-500. DOI: 10.1016/j.biotechadv.2008.05.007.

6. Tothill IE. Biosensors for cancer markers diagnosis. Seminars in Cell \& Developmental Biology. 2009;20(1):55-62. DOI: 10.1016/j.semcdb.2009.01.015.

7. Healy DA, Hayes CJ, Leonard P, McKenna L, O'Kennedy R. Biosensor developments: application to prostate-specific antigen detection. Trends in Biotechnology. 2007;25(3):125-31. DOI:

10.1016/j.tibtech.2007.01.004.

8. Wu J, Fu Z, Yan F, Ju H. Biomedical and clinical applications of immunoassays and immunosensors for tumor markers. Trac-Trends in Analytical Chemistry. 2007;26(7):679-88. DOI: 10.1016/j.trac.2007.05.007.

9. Li Y, Liu X, Lin Z. Recent developments and applications of surface plasmon resonance biosensors for the detection of mycotoxins in foodstuffs. Food Chemistry. 2012;132(3):1549-54. DOI: $10.1016 /$ j.foodchem.2011.10.109.

10. Svabenska E, Kovar D, Krajicek V, Pribyl J, Skladal P. Electrochemical Biosensor for Detection of Bioagents. International Journal of Electrochemical Science. 2011;6(12):5968-79. URL: http://www.electrochemsci.org/papers/vol6/6125968.pdf.

11. Piliarik M, Parova L, Homola J. High-throughput SPR sensor for food safety. Biosensors \& Bioelectronics. 2009;24(5):1399-404. DOI: 10.1016/j.bios.2008.08.012.

12. Mascini M, Tombelli S. Biosensors for biomarkers in medical diagnostics. Biomarkers. 2008;13(7-8):637-57. DOI: 10.1080/13547500802645905.

13. Eicher D, Merten CA. Microfluidic devices for diagnostic applications. Expert Review of Molecular Diagnostics. $2011 ; 11(5)$ :505-19. DOI: $10.1586 / \mathrm{erm} .11 .25$

14. Gervais L, de Rooij N, Delamarche E. Microfluidic Chips for Point-of-Care Immunodiagnostics. Advanced Materials. 2011;23(24):H151-H76. DOI: 10.1002/adma.201100464.

15. Trietsch SJ, Hankemeier T, van der Linden HJ. Lab-on-a-chip technologies for massive parallel data generation in the life sciences: A review. Chemometrics and Intelligent Laboratory Systems. 2011;108(1):64-75. DOI: 10.1016/j.chemolab.2011.03.005.

16. Uludag Y, Sagiroglu M, Ersoy A, Edis A, Budak S, Demiralp A, inventors. An electrochemical sensor array and apparatus, PCT/IB2015/0524792015. URL:

https://patents.google.com/patent/WO2015155665A1/en?q=\%22An+electrochemical+sensor+arr ay+and+apparatus\%22.

17. Olcer Z, Esen E, Muhammad T, Ersoy A, Budak S, Uludag Y. Fast and sensitive detection of mycotoxins in wheat using microfluidics based Real-time Electrochemical Profiling. Biosensors \& Bioelectronics. 2014;62:163-9. DOI: 10.1016/j.bios.2014.06.025.

18. Uludag Y, Olcer Z, Samil Sagiroglu M. Design and characterisation of a thin-film electrode array with shared reference/counter electrodes for electrochemical detection. Biosensors and Bioelectronics. 2014;57(0):85-90. DOI: 10.1016/j.bios.2014.01.048.

19. García-Raya D, Madueño R, Sevilla JM, Blázquez M, Pineda T. Electrochemical characterization of a 1,8-octanedithiol self-assembled monolayer (ODT-SAM) on a Au(l 111 ) single crystal electrode. Electrochimica Acta. 2008;53(27):8026-33. DOI: 10.1016/j.electacta.2008.06.017. 
20. Cavallini M, Bracali M, Aloisi G, Guidelli R. Electrochemical STM investigation of 1,8octanedithiol self-assembled monolayers on $\mathrm{Ag}(111)$ in aqueous solution. Langmuir. 1999;15(8):3003-6. DOI: 10.1021/la9815392.

21. Campuzano S, Pedrero M, Montemayor C, Fatas E, Pingarron JM. Characterization of alkanethiol-self-assembled monolayers-modified gold electrodes by electrochemical impedance spectroscopy. Journal of Electroanalytical Chemistry. 2006;586(1):112-21. DOI: 10.1016/j.jelechem.2005.09.007.

22. Senaratne W, Andruzzi L, Ober CK. Self-assembled monolayers and polymer brushes in biotechnology: Current applications and future perspectives. Biomacromolecules. 2005;6(5):242748. DOI: $10.1021 / \mathrm{bm050180a.}$

23. Campuzano S, Pedrero M, Montemayor C, Fatás E, Pingarrón JM. Characterization of alkanethiol-self-assembled monolayers-modified gold electrodes by electrochemical impedance spectroscopy. Journal of Electroanalytical Chemistry. 2006;586(1):112-21. DOI: 10.1016/j.jelechem.2005.09.007.

24. Yang YJ, Khoo SB. Fabrication of self-assembled monolayer of 8-mercaptoquinoline on polycrystalline gold electrode and its selective catalysis for the reduction of metal ions and the oxidation of biomolecules. Sensors and Actuators B-Chemical. 2004;97(2-3):221-30. DOI: 10.1016/j.snb.2003.08.019.

25. Volpe G, Compagnone D, Draisci R, Palleschi G. 3,3 ',5,5'-tetramethylbenzidine as electrochemical substrate for horseradish peroxidase based enzyme immunoassays. A comparative study. Analyst. 1998;123(6):1303-7. DOI: 10.1039/A800255].

26. Uludag Y, Esen E, Kokturk G, Ozer H, Muhammad T, Olcer Z, et al. Lab-on-a-chip based biosensor for the real-time detection of aflatoxin. Talanta. 2016;160:381-8. DOI: 10.1016/j.talanta.2016.07.060.

27. DelCarlo M, Mascini M. Enzyme immunoassay with amperometric flow-injection analysis using horseradish peroxidase as a label. Application to the determination of polychlorinated biphenyls. Analytica Chimica Acta. 1996;336(1-3):167-74. DOI: 10.1016/S0003-2670(96)00377-7.

28. Fanjul-Bolado P, González-Garćia MaB, Costa-Garćia An. Voltammetric determination of alkaline phosphatase and horseradish peroxidase activity using 3-indoxyl phosphate as substrate: Application to enzyme immunoassay. Talanta. 2004;64(2):452-7. DOI:

10.1016/j.talanta.2004.03.003. 